\title{
DUAL-FREQUENCY OBSERVATIONS OF PULSAR MICROSTRUCTURE FROM PSR $1133+16$
}

\author{
D. R. STINEBRing \\ Physics Department, Princeton University \\ Department of Physics, Oberlin College \\ S. E. ThORSETt AND V. M. KasPI \\ Physics Department, Princeton University
}

\begin{abstract}
We have made pulsar microstructure observations of PSR $1133+16$ at the Arecibo Observatory. Cross-correlation of linearly polarized microstructure between $111 \mathrm{MHz}$ and $318 \mathrm{MHz}$ results in a value of the dispersion measure of $D M=4.8462 \pm 0.0004 \mathrm{pc} \mathrm{cm}^{-3}$ at epoch 1989.9. This value is consistent with the average profile determination of Phillips and Wolszczan (1990) obtained at epoch 1989.2, but not with the microstructure value found by Popov et al. (1987) in early 1980. Time variation of the integrated electron content over this ten-year span is a plausible explanation for the discrepancy.
\end{abstract}

\section{Observations}

We made observations of PSR $1133+16$ on 17 December 1989. We obtained simultaneous data in four frequency bands (a single linear polarization): $111.5 \mathrm{MHz}, 112.0 \mathrm{MHz}, 313.31 \mathrm{MHz}$, and 318.31 MHz. Faraday rotation between $111.5 \mathrm{MHz}$ and $112.5 \mathrm{MHz}$ is only about $10^{\circ}$ (ISM and ionosphere), and similar between 313 and $318 \mathrm{MHz}$, but the total rotation between the $111-\mathrm{MHz}$ and 318 $\mathrm{MHz}$ band is large ( $\sim 4$ turns) and undetermined. A strong single pulse, with a micropulse spike, is shown in figure 1 (no signal was seen at $112.0 \mathrm{MHz}$, and these data were not used subsequently).

\section{Analysis}

We analyzed a 420-pulse sequence obtained in this fashion. We cross-correlated the $111.5-\mathrm{MHz}$ data against both the 313 and $318-\mathrm{MHz}$ data. The cross-correlation function (CCF) between $111 \mathrm{MHz}$ and $313 \mathrm{MHz}$ is shown in figure $2 \mathrm{a}$ with a close-up view in figure $2 b$. The CCF shows a broad subpulse feature ( $\sim 7 \mathrm{~ms}$ width) topped by a micropulse CCF feature with a width of about $1 \mathrm{~ms}$. We estimate the delay peak of the micropulse feature to be $12.4 \pm 0.1 \mathrm{~ms}$, to which must be added an instrumental delay of $1408.300 \mathrm{~ms}$. We determined the dispersion measure $(D M)$ between these two frequencies using

$$
D M^{\prime}=\frac{2.410000 \times 10^{-4}\left(t_{2}-t_{1}\right)}{\left(\nu_{2}^{-2}-\nu_{1}^{-2}\right)} \quad \mathrm{pc} \mathrm{cm}^{-3},
$$

where $\nu_{1}$ and $\nu_{2}$ are in $\mathrm{MHz}$ and $t_{1}$ and $t_{2}$ are in seconds. (The effective center frequencies of
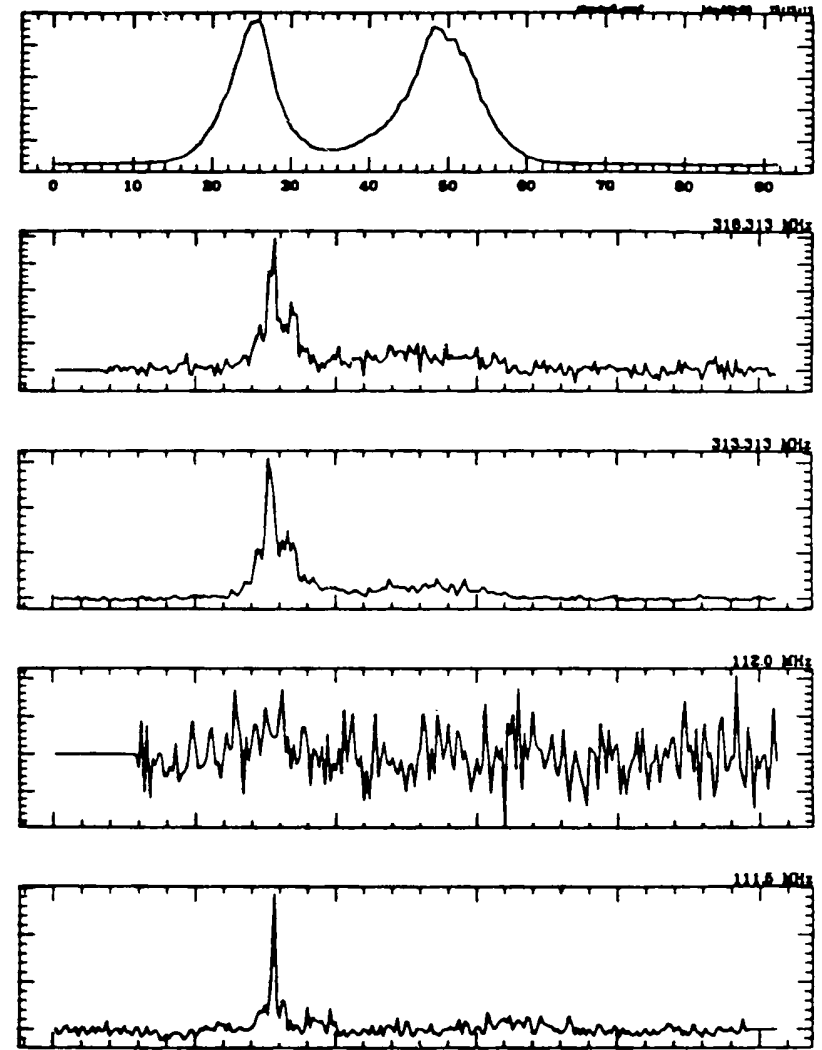

Figure 1 Observations of a single pulse from PSR $1133+16$ observed with the $111 / 318 \mathrm{MHz}$ feed system in December 1989 (P1478). The panels are, from top to bottom: a) $313-\mathrm{MHz}$ average profile, b) $318.3 \mathrm{M} \mathrm{Hz}$, c) $313.3 \mathrm{M} \mathrm{Hz}$, d) $112.0 \mathrm{MHz}$ (no signal present), and e) $111.5 \mathrm{MHz}$. An isolated micropulse is seen in panels $b, c$, and $e$.

the two bands are $111.433 \pm 0.0001$ and $313.000 \pm$ $0.0001 \mathrm{MHz}$ because of the use of coherent dedispersing equipment. We have used the conventional 


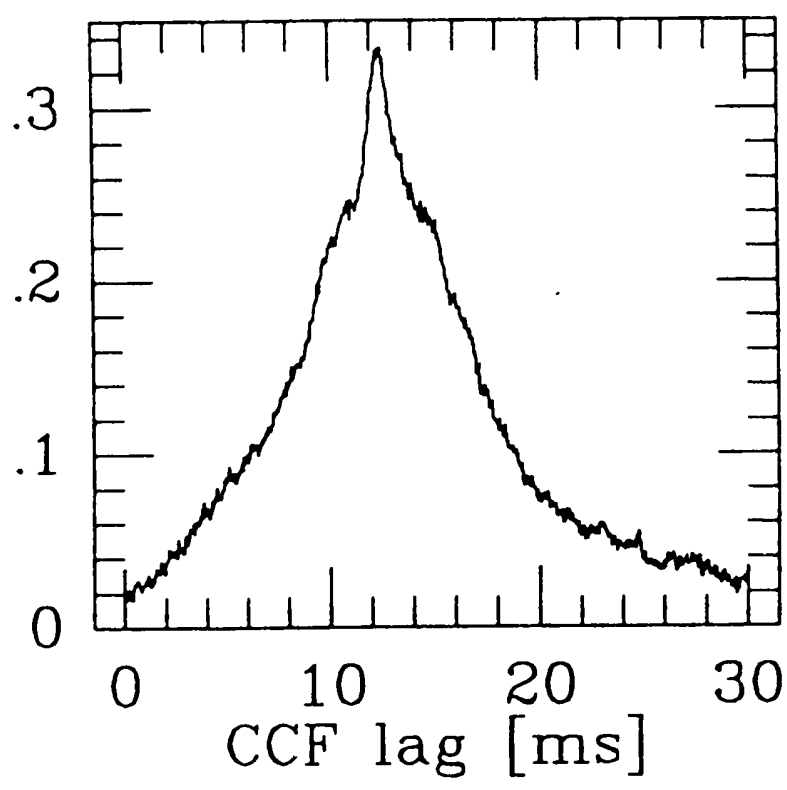

Figure 2a The average cross-correlation function (313 $\mathrm{MHz}$ to $111 \mathrm{MHz}$ ) of many pulses such as those shown in figure 1 . The maximum has a half-width of about $500 \mu \mathrm{s}$, characteristic of the microstructure from this pulsar.

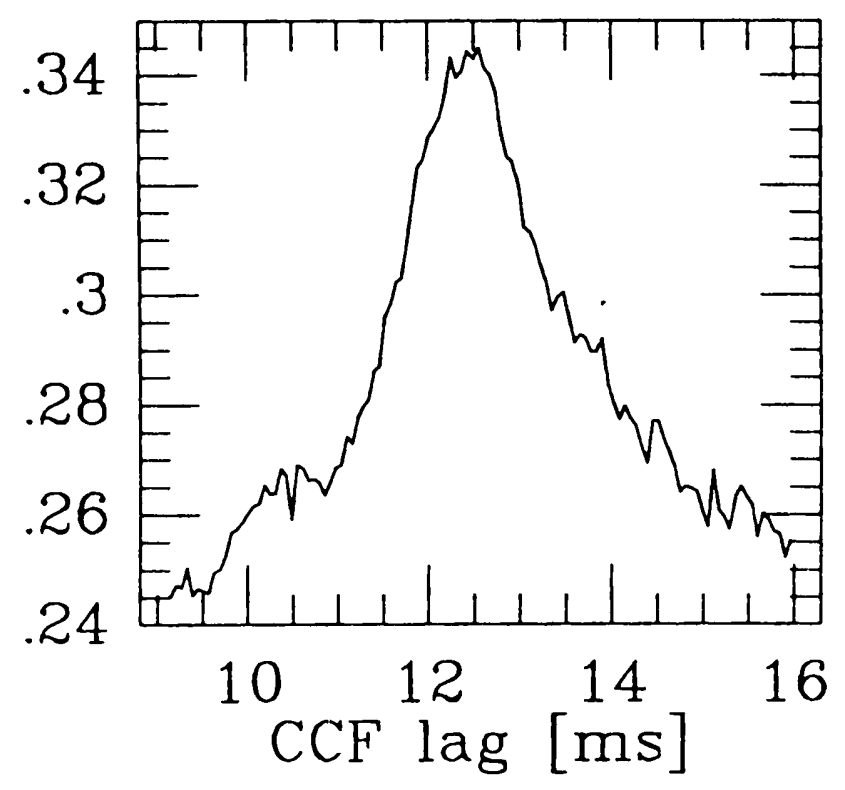

Figure 2b A close-up of the same CCF as shown in figure 2a. The peak was estimated to be at a delay of $12.4 \pm 0.1 \mathrm{~ms}$, to which must be added an instrumental delay of $1408.3 \mathrm{~ms}$.

value of 2.410000 in translating delay into $D . M$; a more precise value, based on the recent SI readjustment of fundamental constants, is 2.41033.)

When corrected for the Doppler shift of the Earth's orbital motion using $D M=D M^{\prime} /(1+\beta)$, where $\beta=v / c$ is positive for motion of the earth toward the pulsar, and the frequencies and times are measured in the topocentric frame, this results in a determination of

$$
D M=4.8463 \pm 0.0004 \mathrm{pc} \mathrm{cm}^{-3}
$$

for the $111.5-\mathrm{MHz}$ to $313.3-\mathrm{MHz}$ CCF. A similar analysis of the $111.5-\mathrm{MHz}$ to $318.3-\mathrm{MHz} \mathrm{CCF}$ yields

$$
D M=4.8461 \pm 0.0004 \mathrm{pc} \mathrm{cm}^{-3} \text {. }
$$

\section{Results}

These two points, determined by the microstructure cross-correlation technique, are plotted in figure 3 at epoch 1989.95. Two other microstructure $D M$ determinations are also shown in figure 3: Boriakoff (private communication), 196 to $318 \mathrm{MHz}$, and Popov, Smirnova, and Soglasnov (1987), 40 to $102 \mathrm{MHz}$. Two $\mathrm{DM}$ determinations obtained by cross-correlating average profiles are also shown: Craft (1973), 40 to $430 \mathrm{MHz}$, and Phillips and Wolszczan (1990), 25 to $2380 \mathrm{MHz}$. Note that the lower point next to the Phillips and Wolszczan label uses a conversion constant of 2.41000 rather than the value they used of $\approx 2.41033$ in reporting $D M=4.8471 \mathrm{pc} \mathrm{cm}^{-3}$. This moves their point into agreement with our microstructure $D M$ determination, obtained less than a year later.

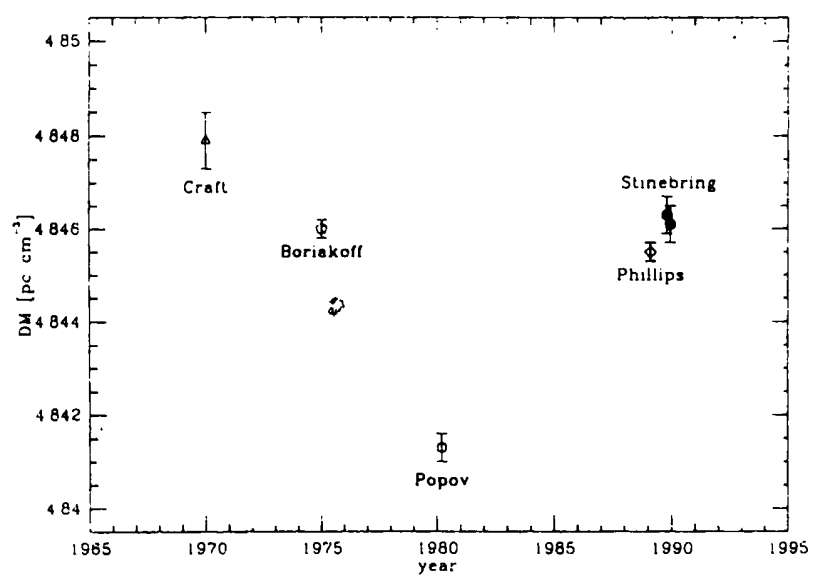

Figure s A comparison of $D M$ determinations for PSR 1133+16 obtained over 20 years using two different techniques. The points labelled Boriakof, Popov, and Stinebring indicate microstructure (single-pulse) crosscorrelations. The Craft and Phillips points represent average profile cross-correlations.

\section{Conclusions}

We are hesitant to draw sweeping (or sweepback, see Shitov et al. 1988) conclusions from these initial results. In particular, we emphasize that our two points are determined from 420 pulses and that neither of two other blocks of pulses observed during the same hour-long session show microstructure CCF features. We are also concerned about the use of linearly polarized feeds at both observing bands. With these caveats in mind, however, we make the following conclusions: 
1. Our microstructure $D M$ determination is $0.001 \mathrm{pc} \mathrm{cm}^{-3}$ per year are seen in seven years of identical to the average profile determination of dual-frequency timing data from the millisecond Phillips and Wolszczan (1990) obtained at about pulsar, PSR 1937+21 (Rawley, Taylor, and Davis the same epoch.

2. Our $D M$ determination is consistent with Boriakoff's 1975 microstructure determination, but not with Popov's extensive low-frequency measurements in 1980.

A discrepancy at this level of precision could be due to many things (e.g., use of different constants, small observing frequency errors, a frequency dependence of microstructure $D M$ determinations), and the large amount of time that has elapsed since the earlier observations makes it unlikely that a definitive explanation will be found. A time variation of the integrated electron content $(D M)$ along the line of sight is another plausible 1988, Stinebring et al. 1990). Although the path length to PSR $1937+21(D M=71.04)$ is much longer than that to PSR $1133+16$, the absolute error should only depend only on $D M^{1 / 2}$ if the fluctuations are caused by random irregularities in the ISM.

This tabulation of $D M$ values for PSR $1133+16$ raises more questions than it answers. But the importance of determining high precision $D M$ values using both microstructure and profile alignment techniques at the same epoch is clear from this study. We have embarked on such a program and hope to be able to clarify what is being measured when we measure $D M$. 